\title{
Circumstances affecting the protection against electrode potential rise (EPR)
}

\author{
J. Ladányi, Gy. Varjú \\ Department of Electric Power Engineering, Power Systems and Environment Group \\ Budapest University of Technology and Economics \\ Egry József u. 18, 2nd floor, H-1111 Budapest (Hungary) \\ Phone number: +0036 1 4633021, fax: +0036 1 4633013, e-mail: ladanyi.jozsef@vet.bme.hu
}

\begin{abstract}
In this paper the earthing resistance of substations with different earth electrode and soil structures is investigated by simulation studies with the use of CDEGS (Current Distribution, Electromagnetic Fields, Grounding and Soil Structure Analysis) software code. The paper presents the effect of grid geometry, soil characteristics, the effectiveness of the vertical earth rods and the conductor diameter.
\end{abstract}

\section{Keywords}

Earthing grid, substation, earthing resistance, simulation

\section{Introduction}

Required power of the big cities keeps growing year by year. It is necessary to establish new high voltage (HV) / medium voltage (MV) transformer stations to satisfy power demand in densely populated urban areas. The area available for installing $\mathrm{HV} / \mathrm{MV}$ substation is restricted and becomes more and more expensive in the city. Considering this condition earthing resistance of the substations must be low enough to satisfy safety (step voltage, touch voltage, earth potential rise) and electromagnetic compatibility requirements (earth potential rise, potential difference inside the station affecting the secondary wiring).

Simulation study has been done to assess the effect of the following circumstances affecting the earthing resistance:

- Geometrical dimensions of substation earthing system

- $\quad$ Mesh size of the grid
- Application of the driven vertical rod electrodes (deep earth rods)

- Soil characteristics (value of the specific resistivity and stratified structure)

In case of simply grid arrangements the earthing resistance can be determined with analytical expressions given in the technical literatures [1] [2]. Nowadays the earthing resistance of complex earthing systems with the consideration of the actual soil resistivity values and structures of stratified earth can also be calculated by sophisticated computer codes based on numerical techniques [3].

In this paper the effects of the above listed conditions on the earthing resistance are investigated and the relative importance of the different parameters are evaluated.

\section{Simulation technique}

The resistance of an investigated earthing system has been calculated, by definition, as the ratio of the earth potential rise (EPR) and the current causing it (Ohm's law). That is why current injection technique has been used. Thus, a test current has been injected into the grid, which has caused a potential rise on the grid. The ratio of the potential rise to the remote earth at the current injection point and to the current value gives the earthing resistance.

Injected current in all cases is $10 \mathrm{kA}$ with zero phase angle. Injection point was placed on the center of the grid except when the grid is a single frame where it is one of a corner points. 


\section{Examined variations}

Earthing resistance depends on above-mentioned circumstances.

In this paper the following effects were studied:

- Total length of conductors (mesh size of theirs)

- $\quad$ Diameter of the conductors

- Soil structure, uniform and two layered

- Value of the soil resistivity

- Application of driven vertical rod electrodes

- Theirs combinations

In addition the electrode potential rise (EPR) was also investigated.

The area of earthing system of substations installed in densely populated urban areas is generally about $400 \mathrm{~m}^{2}$. Accordingly a quadratic, $400 \mathrm{~m}^{2}$ earthing grid during is used the simulations (Fig. 1.).

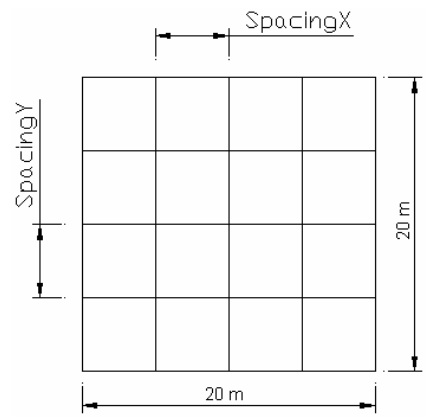

Fig. 1. Mesh size of the grid

TABLE I. - Examined cases of the mesh size of grid $\left(\rho_{\text {soil }}=100 \Omega \mathrm{m}\right)$

\begin{tabular}{|c|c|c|c|c|c|}
\hline \multicolumn{2}{|c|}{ Conductor } & \multicolumn{2}{c|}{ Grid } & \multicolumn{2}{c|}{ Earthing resistance } \\
\hline $\begin{array}{c}\text { Total } \\
\text { length } \\
{[\mathrm{m}]}\end{array}$ & $\begin{array}{c}\text { Total } \\
\text { surface } \\
{\left[\mathrm{m}^{2}\right]}\end{array}$ & $\begin{array}{c}\text { Spacing } \\
\mathrm{X} \\
{[\mathrm{m}]}\end{array}$ & $\begin{array}{c}\text { Spacing } \\
\mathrm{Y} \\
{[\mathrm{m}]}\end{array}$ & {$[\Omega]$} & p.u. \\
\hline 80 & 5.03 & 20 & 20 & 2.76 & 1.24 \\
\hline 100 & 6.28 & 20 & 10 & 2.56 & 1.15 \\
\hline 120 & 7.54 & 10 & 10 & 2.44 & 1.10 \\
\hline 140 & 8.80 & 20 & 5 & 2.35 & 1.06 \\
\hline 160 & 10.05 & 10 & 5 & 2.30 & 1.03 \\
\hline 200 & 12.57 & 5 & 5 & 2.22 & 1.00 \\
\hline 220 & 13.82 & 20 & 2.5 & 2.21 & 0.99 \\
\hline 240 & 15.08 & 10 & 2.5 & 2.18 & 0.98 \\
\hline 280 & 17.59 & 5 & 2.5 & 2.15 & 0.97 \\
\hline 360 & 22.62 & 2.5 & 2.5 & 2.11 & 0.95 \\
\hline 460 & 28.90 & 20 & 1 & 2.13 & 0.96 \\
\hline 480 & 30.16 & 10 & 1 & 2.09 & 0.94 \\
\hline 520 & 32.67 & 5 & 1 & 2.06 & 0.93 \\
\hline 600 & 37.70 & 2.5 & 1 & 2.04 & 0.92 \\
\hline 840 & 52.78 & 1 & 1 & 2.02 & 0.91 \\
\hline
\end{tabular}

Considering this size it is a special problem to keep earthing resistance low enough value. The examined cases concerning the grid structure and mesh sizes are given in Table 1 . In all cases the grid depth is $0.8 \mathrm{~m}$.

\section{A. Soil characteristic}

From the point of view of earthing resistance the soil resistivity, stratification, thickness of layers, number of layers are determinant.

Two cases were examined, on the one hand the soil is uniform and the other hand it was a two-layer soil (Fig.2.)

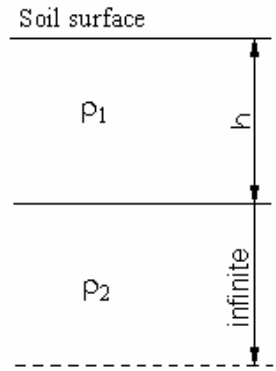

Fig. 2. Model of two-layer soil

\section{Presentation of the results}

The earthing resistance obtained from the simulations are usually plotted vs. the total length of conductors.

Earthing resistance as a functions of total length of conductors is shown in the Fig. 3.

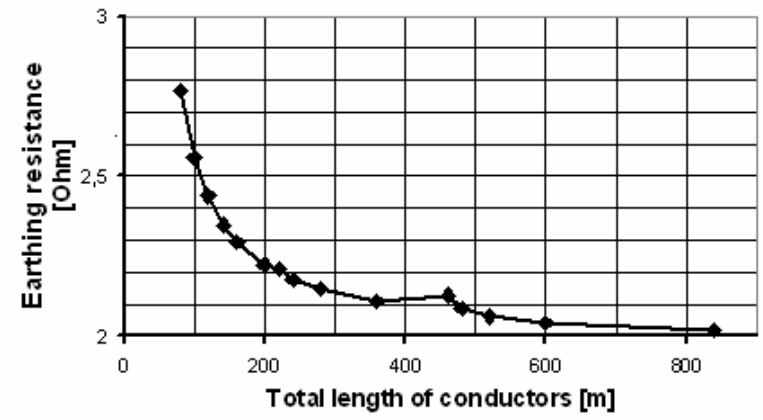

Fig. 3. Earthing resistance vs. conductor length ( $\rho_{\text {soil }}=100 \Omega \mathrm{m}$, uniform soil)

A good earthing system provides a low resistance to remote earth in order to minimize the EPR. For most transmission and other large substations, the ground resistance is usually about $1 \Omega$ or less. In smaller distribution substations, the usually acceptable range is from $1 \Omega$ to $5 \Omega$, depending on the local conditions [2].

In this case, the highest value also is less than 2.8 $\Omega$, even if the "grid" is just a frame. 
Small mesh size of the conductors causes that the establishment cost becomes more expensive. Therefore in the practice, the average mesh size of conductors is about $3 \mathrm{~m}$ to $6 \mathrm{~m}$. Considering this fact the earthing resistance is about $2.22 \Omega$ at 5 by 5 m mesh size.

Regarding the effect of the change of conductor diameter, the total surface is growing with the increase of the diameter which causes a decrease in the earthing resistance. If the diameter is doubled the earthing resistance changes less than $5 \%$. The material of the grid does not influence the earthing resistance [4.].

Earthing resistance is directly proportional to soil resistivity in that case of uniform soil while this relation is non-linear when the soil is non-uniform (e.g. stratified). This non-linear function is presented on the Fig. 4. $\rho_{1}$ and $\rho_{2}$ are the resistivity of the upper and the lower layers, respectively. Thickness (h) of the upper layer is $5 \mathrm{~m}$ while the lower is infinite in depth. Parameter is the upper layer resistivity.

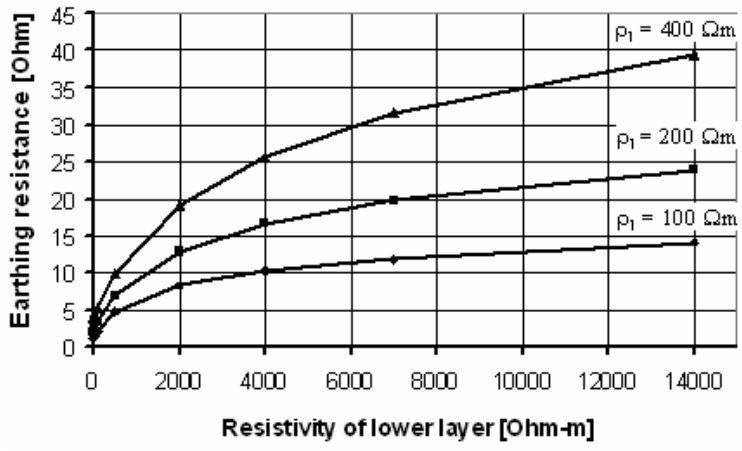

Fig. 4. Earthing resistance vs. the resistivity of the bottom layer, Parameter the resistivity of the upper layer (total length of conductors is $200 \mathrm{~m}$ )

Another option is that case when the upper layer has a lower resistivity than the bottom layer. It is shown on the Fig. 5.

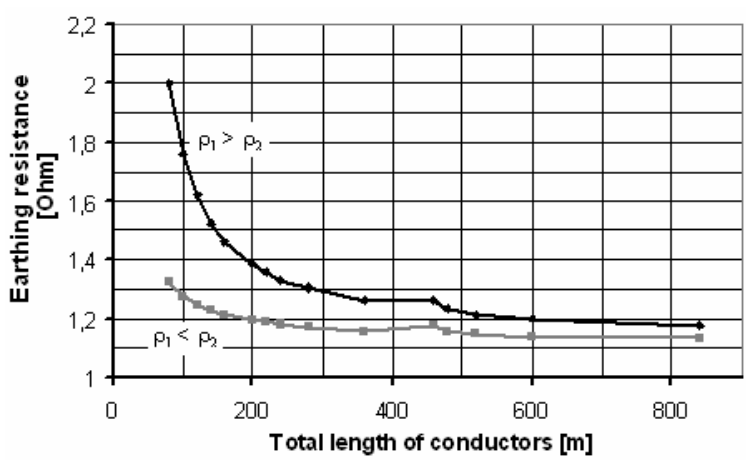

Fig. 5. Earthing resistance vs. conductor length. Parameter: ratio of the resistivities of the two layers, i.e. 30 and $100 \Omega \mathrm{m}$

As it can be seen on the figure it is favourable if the top layer has lower resistivity.

\section{B. Vertical earth rods}

Accordingly to the standards concentrated earthing have to apply for the surge arresters and the star point of the transformer(s) in the transformer stations. It can be vertical earth rod. The effect of such rods has also been investigated.

The earth rods were placed at the center and the four corners of earthing grid except when the grid was only a frame then, of course, no rod was applied at the centre. The length each rod is $10 \mathrm{~m}$.

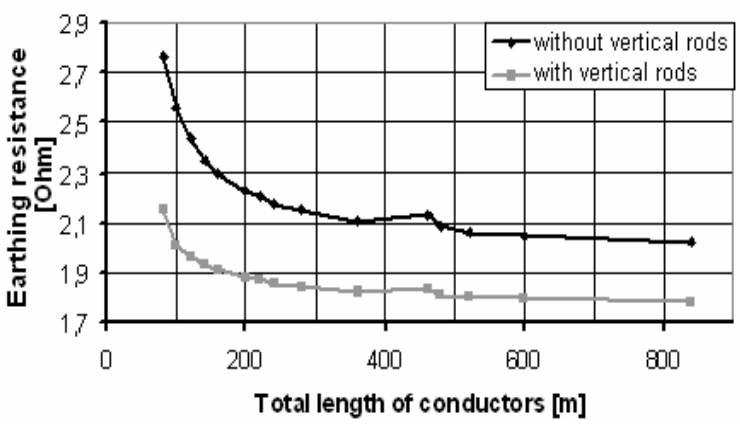

Fig. 6. Earthing resistance vs. conductor length Parameter: application of vertical rod electrodes (Uniform soil: $\rho_{\text {soil }}=100 \Omega \mathrm{m}$ )

When the soil is uniform, the earthing resistance decreases due to the application of the vertical earth rods. This effect is presented on the Fig. 6. The difference between cases is about $10 \%$.

The uniform soil is a theoretical model because in the most practical cases the soil is usually stratified, non-homogeneous medium.

The earthing resistance curves in that case when the soil is two layered is shown in Fig. 7. The difference between the earthing resistance of the grid with and without vertical earth rods is more than in the above-mentioned case. Vertical earth rods make a "direct" contact between layers, thus resulting in lower earthing resistance.

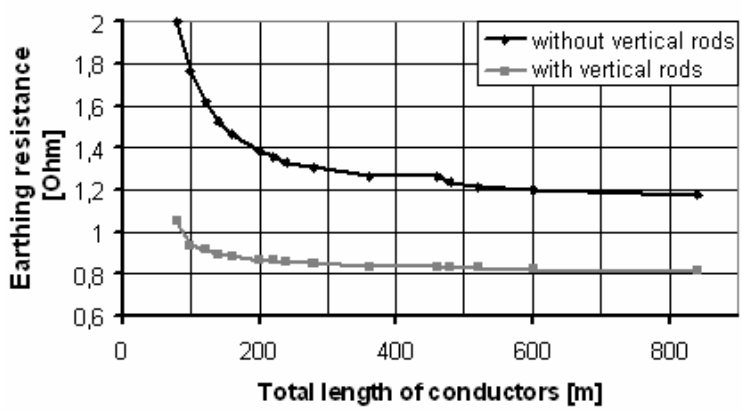

Fig. 7. Earthing resistance vs. conductor length Parameter: application of vertical rod electrodes

(Two-layer soil: $\rho_{1}=100 \Omega \mathrm{m}, \rho_{2}=30 \Omega \mathrm{m}$ )

That case when resistance of the top layer is lower than the bottom layer is presented in Fig. 8. 


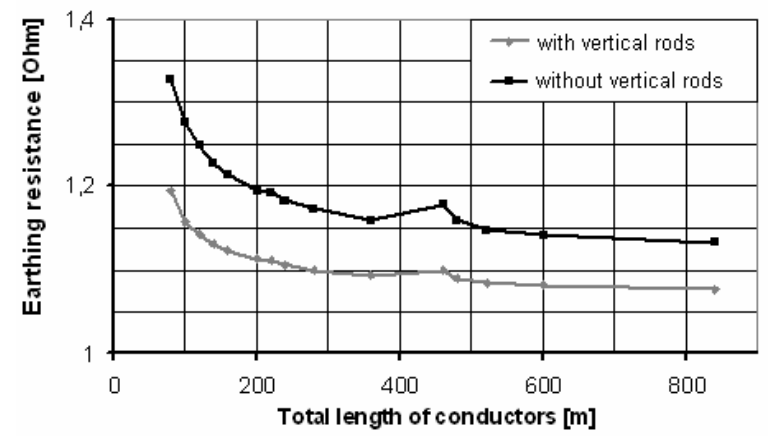

Fig. 8. Earthing resistance vs. conductor length Parameter: application of vertical rod electrodes (Two-layer soil: $\rho_{1}=30 \Omega \mathrm{m}, \rho_{2}=100 \Omega \mathrm{m}$ )
$100 \Omega \mathrm{m}$ resistivity in all cases (Fig. 9. b) and e) ). The changing of potential cone and EPR can be seen in the first row when $\rho_{1}$ is constant and in the second row when $\rho_{2}$.

The center of the earthing grid (mesh size: 5 by 5 $\mathrm{m}$, basic area: $400 \mathrm{~m}^{2}$ ) is in the 0 point. From the results it can be drown that especially disadvantageous that case when the upper layer has the smaller resistivity than the bottom layer.

The maximum EPR values are shown in Fig. 10. The reference value is $2,4 \mathrm{kV}$ which belongs to the uniform soil $(\rho=100 \Omega m)$.
This soil structure results in significantly lower earth resistance even without the earthing rods. Or another words, the improvement in the earthing resistance due to the rod electrodes is much less than in that case when the bottom layer has the smaller resistivity.

\section{Earth potential rise (EPR)}

Different soil structures causes different earth potential rise. In addition decrement of the potential cone depends on soil structure. Two cases were investigated: on the one hand the soil is two layered and the bottom layer has constant resistivity, and the other hand the top layer has constant resistivity. The reference value of EPR and the reference shape of potential cone belongs to the uniform soil with

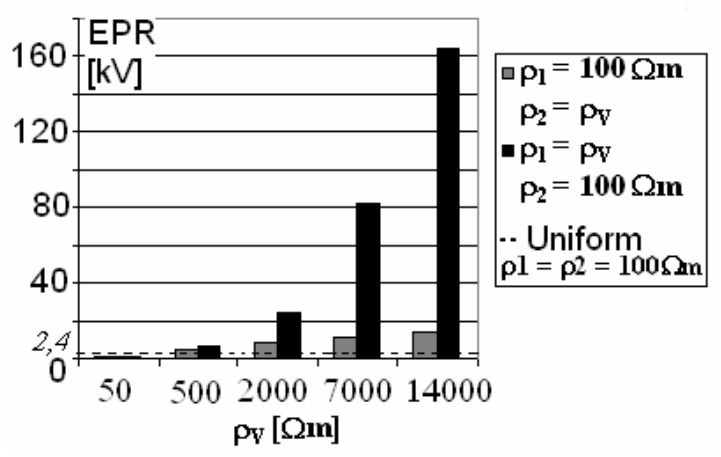

Fig. 10. EPR values in different cases.

Changing of wideness of the potential cone is plotted in Fig. 11. The reference value is $134,5 \mathrm{~m}$ which belongs to the uniform soil ( $\rho=100 \Omega m)$. 


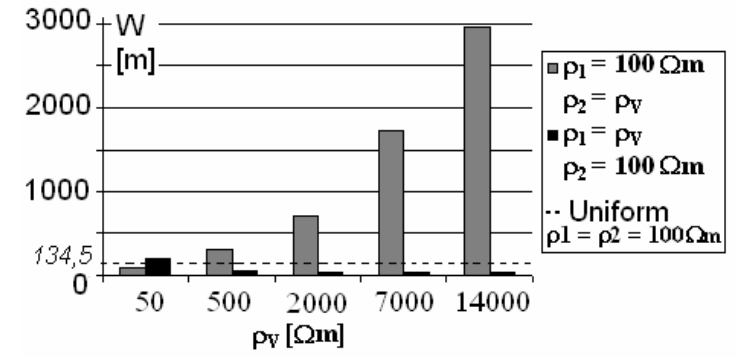

Fig. 11. Wideness of potential cone at $10 \%$ of maximum EPR values.

\section{Investigation of not conventional arrangement}

Nowadays, as it has been mentioned in the introduction, the area available for installing HV/MV substation is restricted. Therefore it is a real possibility to built underground substation in the densely populated areas. The earthing system of such a not conventional underground 120/10 kV substation is investigated in the followings.

The basic arrangement of the earthing system is plotted in Fig. 12. On the top $(-0.5 \mathrm{~m})$ there is a frame which is connected to the vertical rods. The earthing grid itself is at the bottom $(-18.5 \mathrm{~m})$.

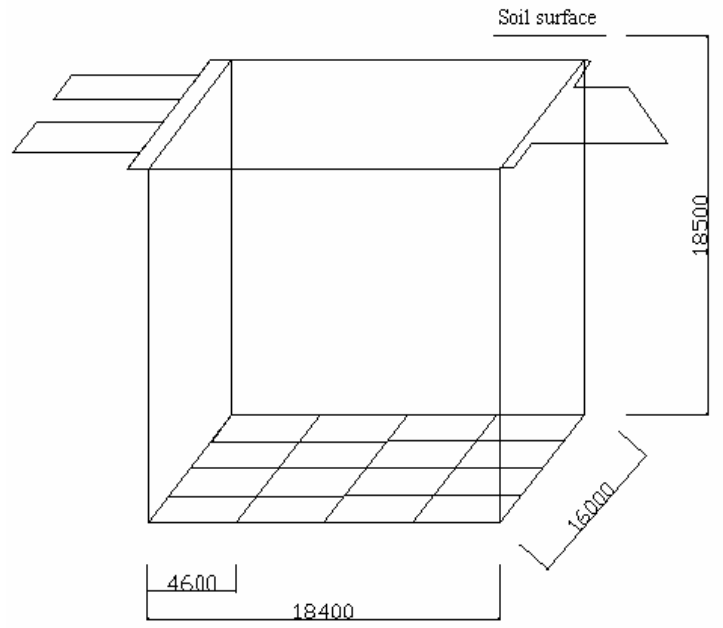

Fig. 12. Arrangement of the earthing system

Three cases were examined:

(1) The earthing grid and the vertical rods are embedded in concrete and inside area of the substation is filled with soil.

(2) The earthing grid and the vertical rods embedded in concrete and inside area of the substation is filled with air

(3) The earthing grid embedded in concrete and inside area of the substation is filled with air

Basic parameters are the followings:
- Uniform soil with $20 \Omega$ m resistivity

- Resistivity of the concrete is $60 \Omega \mathrm{m}$ (from literature)

- Injected current is $1000 \mathrm{~A}$ into the center of the grid

- Air resistivity is $1 \mathrm{E}+18 \Omega \mathrm{m}$ (homogenous)

- Material of earthing system is $\varnothing 30$ mm cylindrical stainless steel

The results of the simulation study are given in Table 2.

TABLE II. - Result of the simulation study

\begin{tabular}{|c|c|}
\hline Case & Earthing resistance $[\Omega]$ \\
\hline 1. & 0.31 \\
\hline 2. & 0.30 \\
\hline 3. & 0.27 \\
\hline
\end{tabular}

Considering the circumstances the second case is close to the reality. In this case the effect of conductor radius was examined and it is presented in the Table 3.

As a next step the value of the earthing resistance has been compared for conductors having diameters of 20,25 or $30 \mathrm{~mm}$ (Table 3). There are no significant differences between the values. Finally stainless steel conductor has been selected on the bases of different practical considerations.

TABLE III. - Effect of the conductor diameter

\begin{tabular}{|c|c|}
\hline Conductor diameter & Earthing resistance $[\Omega]$ \\
\hline$\varnothing 30 \mathrm{~mm}$ & 0,296 \\
\hline$\varnothing 25 \mathrm{~mm}$ & 0,299 \\
\hline$\varnothing 20 \mathrm{~mm}$ & 0,305 \\
\hline
\end{tabular}

It is worth mentioning that the earthing resistance has been checked by site measurement after the completion of the earthing system. The measured earthing resistance is $R_{f}=0.145 \Omega$. ( $f=50 \mathrm{~Hz}$ ). The difference between simulated and measured value is caused by the soil resistivity.

\section{Conclusion}

The earthing resistance can be determined with analytical expressions given in the technical literature in case of simply grid arrangements for homogenous soil structure.[1][2].

In this paper sophisticated computer codes based on numerical techniques [3] are applied for the investigation of the earthing resistance of complex earthing systems with the consideration of the different soil resistivity values and stratified earth. 
Simulation study has been done to assess the effect of the following circumstances affecting the earthing resistance: geometrical dimensions of substation earthing system, mesh size of the grid, application of the driven vertical rod electrodes, soil characteristics (value of the specific resistivity and stratified structure) and finally the earthing resistance of an unconventional earthing structure.

From the numerical results obtained, the following main conclusions can be drown:

- The decrease of the mesh size of the grid results in significant decrease in the earthing resistance up to size 5 by $5 \mathrm{~m}$.

- In case of stratified soil structure the earthing resistance does not change anymore proportionally to the resistivity of the soil.

- The application of vertical rods improves the earthing resistance significantly only in that case when the resistivity of the lower layer is the smaller one.

- In case of two-layered soil with low resistivity upper layer (wet organic soil), and very high resistivity bottom layer (bedrock) the earthing resistance is essentially determined by the upper layer.
- The diameter of the conductor constituting the grid has of minor effect on the magnitude of the earthing resistance.

- EPR and wideness of the potential cone depends on soil structure.

- In that case when the resistivity of the bottom layer is constant and the resistivity of the top layer is increasing, the maximum value of EPR changes approximately proportionally to the resistivity of the upper layer.

- In the opposite case wideness of the potential cone grows wider with increasing of the bottom layer resistivity.

\section{References}

[1] G.F. Tagg, Earth resistances, George Newnes Limited, London (1964)

[2] IEEE Standard 80-2000, IEEE Guide for Safety in AC Substation Grounding (2000)

[3] Current Distribution, Electromagnetic Fields, Grounding and Soil Structure Analysis (CDEGS) Software package manuals, Safe Engineering Services \& technoligies Ltd., Montreal, Quebec, Canada. Application Version 13.

[4] J. Ladányi, Examination of the effect of the earthing grid material in high voltage substations, M.Sc. thesis, Budapest (2006), (in Hungarian) 